\title{
Properties of optical microsensor based on observation of whispering-gallery modes
}

\author{
V.V. Datsyuk \\ Department of Physics, Kyiv National Taras Shevchenko University, 01033 Kyiv, Ukraine \\ Phone: +380 (44) 2664477, E-mail: datsyuk@univ.kiev.ua
}

\begin{abstract}
Formulae for the intensity of the resonant spontaneous electric-dipole emission are derived in the framework of classical electrodynamics and quantum optics making allowance for inhomogeneous light absorption. Using these formulas, the physical backgrounds of a micrometer-sized optical sensor exploiting the properties of the whispering-gallery modes (WGMs) are studied. The role of the evanescent WGM's field in the considered optical effects is examined.
\end{abstract}

Keywords: whispering-gallery mode, morphology-dependent resonance, microlaser

Paper received 14.02.04; accepted for publication 30.03.04.

\section{Introduction}

Recent publications have showed that spherical micrometer sized-optical cavities can have applications in diverse fields including detection of trace gases [1] and protein [2], automobile industry [3], basic research of photosynthesis [4] and quantum informatics [5]. Applicability of a lasing microsphere as a probe of a near-field scanning optical microscope has been discussed [6].

The main goal of this article is to study the physical backgrounds of application of a WGM microsensor and evaluate its ultimate characteristics.

\section{Spectral indications of the whispering gallery modes}

The whispering gallery modes exist in cavities having spherical or cylindrical symmetry. These surface modes are commonly imagined as closed-trajectory rays confined within the cavity by almost total internal reflections from the surface. Excitation of WGMs causes appearance of the morphology-dependent resonances (MDRs) in spectra of spontaneous emission and lasing, elastic and inelastic light scattering (including fluorescence and Raman scattering) [7, 8]. Thus, the molecular emission spectra, smooth in a bulk volume, exhibit ripple structure when light is radiated from a spherical particle (see Fig. 1). This paper proposes to use a measurement of the MDR intensity for diagnostic of the medium that surrounds optical microsensor.

\section{Effect of light absorption on resonant spon- taneous emission of a cavity}

\subsection{Empirical data}

As a result of generalization of a large number of empirical data it was established the dependence of the spectrally integrated MDR intensity I on light absorption $[9,10]$

$\frac{I}{I_{0}}=\frac{Q_{a}}{Q_{0}+Q_{a}}$

In Eq. (1), the relative intensity is expressed via the quality factor of transparent sphere $Q_{0}$ determined at $m_{i}=0$ and the absorption factor

$Q_{a}=\frac{m_{r}}{2 m_{i}}$

Hereinafter, $m_{r}$ and $m_{i}$ are the imaginary and real part of the complex refractive index $m=m_{r}+i m_{i}$. The value of $Q_{a}$ can be expressed via the light absorption coefficient

$\alpha, Q_{a} \equiv \frac{\omega m_{r}}{c \alpha}$, where $\omega, c$ are the angular frequency and 


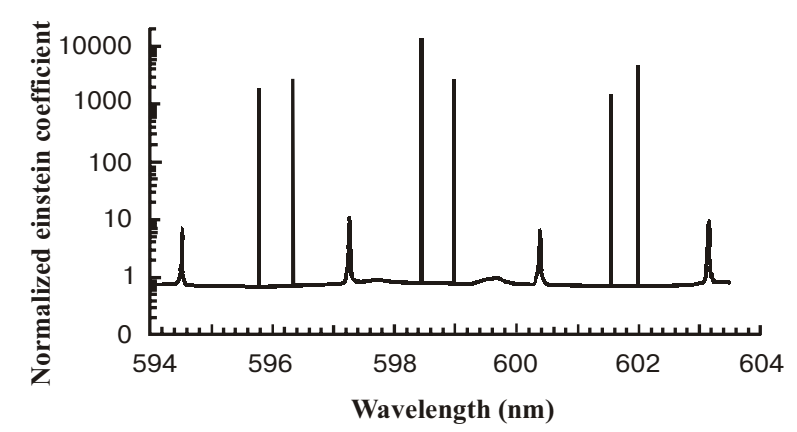

Fig. 1. Normalized Einstein coefficient for spontaneous emission calculated by formulas of Sec. 3.3 for a $15.306 \mu \mathrm{m}$ diameter ethanol droplet.

the velocity of light in vacuum, correspondingly, $m_{r}=$ $=m_{r 3} / m_{r 1}$ is the relative refractive index of volume 3 of a sphere of Fig. 2. The dependence (1) is shown in Fig. 3. In particular, Eq. (1) was proved by observations of resonant emission of $15-\mu \mathrm{m}$-diameter Rhodamine $6 \mathrm{G} /$ ethanol solution droplets with additive light-absorbent nigrosin [9]. For the observed MDRs of several orders, the quantity of $Q_{0}$ lied in the range from $10^{8}$ to $2 \cdot 10^{3}$. At the same time, the factor $Q_{a}$ was varied from $10^{9}$ to $10^{5}$. Particularly, Fig. 3 shows the relative intensity measured for a mode.

\subsection{Asymptotic formula for emission of arbitrary cavity}

In the previous paper [11], the classical electrodynamics has been applied to derive a formula for the rate of spontaneous emission of molecules and atoms in an arbitrary open cavity in the weak coupling approximation with allowance made for homogeneous light absorption or amplification by the cavity material. The formula has been obtained by use of decomposition of the electric field by the modes of high- $Q$ cavity neglecting an influence of $m_{i}$ on the mode structure. This theory yields formula (1) for the intensity of the resonant spontaneous emission.

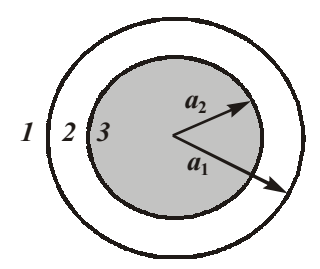

Fig. 2. Scheme of the working part of a spherical microsensor. Index denotes transparent dye-doped dielectric, 1 and 2 are the surrounding medium with possibly different $m_{i}$ and equal $m_{r}$, $a_{1}=a_{2} m_{r 3} / m_{r 1}$.

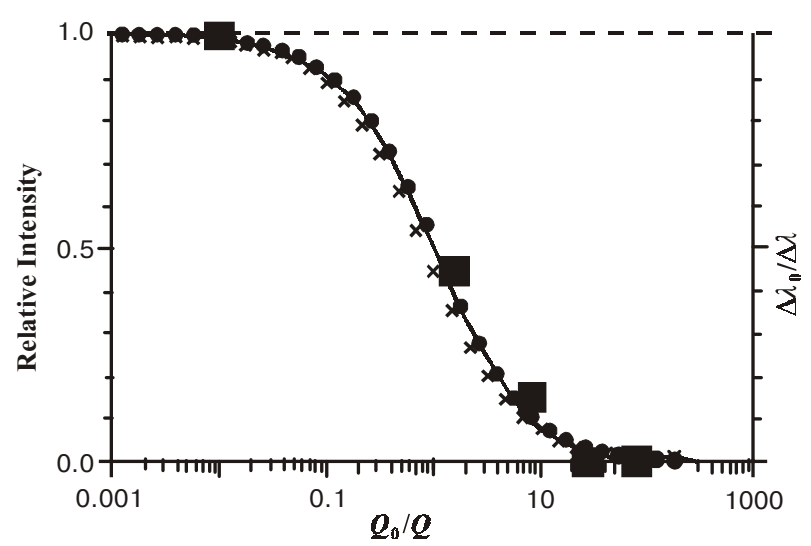

Fig. 3. The relative spontaneous emission intensity as a function of absorption. Solid curve presents the empirical dependence (1) with factor (3), squares indicate the measured data from Ref. [9]. Circles and crosses show the correspondent calculated ratios (13) for absorption in volume 3 and outer layer 2, respectively.

The theoretical model of Ref. [11] can be extended by consideration of not-uniform light-absorbent inclusions in the cavity or surrounding medium. Neglecting the inter-mode energy exchange, the effect of light absorption (or amplification) can be taken into account by modification of the partial $Q_{a}$ factor of the cavity. Namely, the $Q_{a}$ factor has to be determined as follows

$Q_{a}=\frac{\iiint \varepsilon_{i}(\vec{r})\left|\vec{E}_{s}(\vec{r})\right|^{2} d V}{\iiint \varepsilon_{r}(\vec{r})\left|\vec{E}_{s}(\vec{r})\right|^{2} d V}$,

where $\varepsilon_{i}, \varepsilon_{r}$ are the imaginary and real part of the permittivity, respectively, $\vec{E}_{S}(\vec{r})$ is the electric field of the resonant mode. Note that even in the case of a homogeneous distribution of the absorption inside a spherical cavity, Eq. (3) gives a value of $Q_{a}$ that differs from factor (2) well-known in the literature [10],

$\frac{1}{Q_{a}}=\vartheta \frac{2 m_{i}}{m_{r}}, \quad \vartheta<1$.

For example, for calculations of Fig. 3 the factor has been found to be equal to 0.978 and 0.042 for internal and external absorption, respectively. This distinction appears due to the evanescent part of a WGM that gives a small but noticeable contribution to the denominator of the right hand side of Eq. (3). The evanescent field can also provide a dependence of the MDR intensity on the light absorption (or amplification) beyond the microcavity surface. This article proposes to use this effect to develop a new generation of microsensors.

\subsection{Exact calculation of emission intensity for an ideal three-layered spherical structure}

The quantum theory of spontaneous emission in absorbing media [12-14] uses the relation between the Einstein coefficient for spontaneous emission $\mathbf{A}$ and the Green ten- 


\section{V.V. Datsyuk: Properties of optical microsensor based on observation ...}

sor of the Maxwell wave equation. Using Ref. [13], the following equation allowing to calculate the rate of spontaneous emission, averaged over dipole orientation, in a three-layered spherical structure can be obtained,

$$
\begin{aligned}
& \frac{\mathbf{A}}{A_{h}}=\frac{1}{3}+\frac{1}{2} \Re\left\{\frac { 1 } { z _ { 5 } \xi } \sum _ { l = 1 } ^ { \infty } \left[C_{0 l}(2 l+1) \psi_{l}^{2}\left(z_{5} \xi\right)+\right.\right. \\
& \left.\left.+C_{1 l}\left((l+1) \psi_{l-1}^{2}\left(z_{5} \xi\right)+l \psi_{l+1}^{2}\left(z_{5} \xi\right)\right)\right]\right\}
\end{aligned}
$$

where $A_{h}$ is the Einstein coefficient for spontaneous emission in a homogeneous medium with the same $m, \xi=$ $=r_{d} l a_{2}, r_{d}$ is a distance of excited molecule from the center of the sphere, $\psi_{l}(y)$ is the $l$-th order Riccati-Bessel function of the argument $y, z_{2 f} \equiv k_{f} a_{f}, z_{2 f+1} \equiv k_{f+1} a_{f}, a_{f}$ are the sphere radii according to Fig. 2,

$C_{p l}=\frac{T_{\mathrm{F} 2}^{p l}}{T_{\mathrm{P} 2}^{p l}} \frac{T_{\mathrm{P} 1}^{p l} R_{\mathrm{P} 2}^{p l}+T_{\mathrm{F} 1}^{p l} R_{\mathrm{P} 1}^{p l}}{T_{\mathrm{P} 1}^{p l}+T_{\mathrm{F} 1}^{p l} R_{\mathrm{P} 1}^{p l} R_{\mathrm{F} 2}^{p l}}$

index $p=0$ and $p=1$ denotes the transverse electic (TE) and transverse magnetic (TM) modes, respectively,

$$
\begin{aligned}
& R_{P f}^{p l}= \\
& =\frac{k_{f+1-p} \zeta_{l}\left(z_{2 f}\right) \zeta_{l}^{\prime}\left(z_{2 f+1}\right)-k_{f+p} \zeta_{l}^{\prime}\left(z_{2 f}\right) \zeta_{l}\left(z_{2 f+1}\right)}{k_{f+1-p} \psi_{l}\left(z_{2 f}\right) \zeta_{l}^{\prime}\left(z_{2 f+1}\right)-k_{f+p} \psi_{l}^{\prime}\left(z_{2 f}\right) \zeta_{l}\left(z_{2 f+1}\right)},
\end{aligned}
$$

$R_{F f}^{p l}=$

$=\frac{k_{f+1-p} \psi_{l}\left(z_{2 f}\right) \psi_{l}^{\prime}\left(z_{2 f+1}\right)-k_{f+p} \psi_{l}^{\prime}\left(z_{2 f}\right) \psi_{l}\left(z_{2 f+1}\right)}{k_{f+1-p} \zeta_{l}\left(z_{2 f}\right) \psi_{l}^{\prime}\left(z_{2 f+1}\right)-k_{f+p} \zeta_{l}^{\prime}\left(z_{2 f}\right) \psi_{l}\left(z_{2 f+1}\right)}$,

$T_{P f}^{p l}=$

$\frac{k_{f}\left[\psi_{l}\left(z_{2 f+1}\right) \zeta_{l}^{\prime}\left(z_{2 f+1}\right)-\psi_{l}^{\prime}\left(z_{2 f+1}\right) \zeta_{l}\left(z_{2 f+1}\right)\right]}{k_{f+1-p} \psi_{l}\left(z_{2 f}\right) \zeta_{l}^{\prime}\left(z_{2 f+1}\right)-k_{f+p} \psi_{l}^{\prime}\left(z_{2 f}\right) \zeta_{l}\left(z_{2 f+1}\right)}$,

$T_{F f}^{p l}=$

$=\frac{k_{f}\left[\psi_{l}\left(z_{2 f+1}\right) \zeta_{l}^{\prime}\left(z_{2 f+1}\right)-\psi_{l}^{\prime}\left(z_{2 f+1}\right) \zeta_{l}\left(z_{2 f+1}\right)\right]}{k_{f+p} \psi_{l}\left(z_{2 f+1}\right) \zeta_{l}^{\prime}\left(z_{2 f}\right)-k_{f+1-p} \psi_{l}^{\prime}\left(z_{2 f+1}\right) \zeta_{l}\left(z_{2 f}\right)}$,

$\zeta_{l}(y) \equiv \sqrt{\frac{\pi y}{2}} H_{l+1 / 2}^{(1)}(y), H_{l+1 / 2}^{(1)}$ is the Hankel function of the first kind.

In order to reduce a number of input parameters one can average $\mathbf{A}$ over $r_{d}$,

$A=3 \int_{0}^{1} \mathbf{A}(\xi) \xi^{2} d \xi$
The calculation of the integral on the right-hand side of the above equation was executed using the following relation

$$
\int_{0}^{1} \psi_{1}^{2}(z \xi) d \xi=\frac{1}{2} \psi_{l}^{2}(z)\left(1+\frac{1}{p_{l}^{2}}+\frac{2 l+1}{z p_{l}}\right),
$$

where $p_{l} \equiv \psi_{l}(z) / \psi_{l-1}(z)$ is a term incorporated into the algorithm of the computation of $\psi_{l}$ [15].

Even though the formulae of quantum optics for $A$ are known, calculation of the intensity of the resonant spontaneous emission $I$ is not a trivial exercise. A way of determination of $I$ has not been discussed in the quantum theory of spontaneous emission in absorbing media [1214]. To calculate $I$ let us use two assumptions: (i) the rate of spontaneous emission into a resonant mode remains constant [16-12] independently of the mechanism of the broadening of the MDR and (ii) the energy radiated from the cavity is proportional to the part of the mode-energy losses attributed to radiation. Joining these two basic assumptions and presuming that the number of the active molecules is independent of $m_{i}$, one gets the following formula

$\frac{I}{I_{0}}=\frac{\Delta \lambda_{0}}{\Delta \lambda}$

where $\Delta \lambda$ is the FWHM of a MDR, $I=I_{0}$ and $\Delta \lambda=\Delta \lambda_{0}$ at $m_{i}=0$. This article reports the results of calculations of the ratio $\frac{\Delta \lambda_{0}}{\Delta \lambda}$ executed using Eqs. (5)-(13). The calculation was performed for the $\mathrm{TM}_{94}^{2}$ mode observed [9] in spectra of a droplet with a diameter of $2 a=15.306 \mu \mathrm{m}$ and $m_{r}=1.36212$. From Eq. (5), it was found a moderate value of the quality factor $Q_{0}$ of this mode equal to 6.9.106. The same value was calculated by Chylek and co-authors [9] using the Lorents-Mie theory of elastic light scattering.

The knowledge of relation (13) allows to draw one line in Fig. 3 for both internal (see circles) and external (see crosses) light absorption. Note that neighboring circle and crosses in Fig. 3 correspond to values of $m_{i}$ which differ by a factor of 20. Thus, Eq. (13) is in excellent agreement with the empirical data. In the case of internal absorption and the data of Fig. 3, differences of values $I / I_{0}$ calculated by Eq. (13) and those found from Eq. (1) with parameter (3) are less than 0.002 at $Q_{a} / Q_{0}$ varied from 0.2 to 200 .

\section{Characteristics of optical microsensor}

The relation (1) can be used as a base for measurement of the absorption coefficient $\alpha$ in a wide range of $\lambda$. The ratio $I / I_{0}$ is the most sensitive to $\alpha$ in the region of $Q_{0} \approx Q_{a}$ or $\alpha \approx \frac{\omega}{c} m_{r} Q_{0}^{-1}$. The factor $Q_{0}$ is known to depend on the mode indices and can be as large as $10^{9}$ [8]. (Paper 


\section{V.V. Datsyuk: Properties of optical microsensor based on observation ...}

[22] reported an ultra-high value of $Q \approx 10^{12}$.) Since the mode is located in a surface layer of the sensor, I have estimated that a value of $\alpha \approx 10^{-8} \mathrm{~cm}^{-1}$ can be measured in a volume of $10^{-8} \mathrm{~cm}^{-3}$.

\section{Conclusions}

In this paper, the following results have been obtained:

- the formula (1) with factor (3) for the intensity of the resonant spontaneous emission from a cavity allowing for arbitrary distribution of light absorption or amplification in a medium has been grounded. This formula has been applied to calculate the MDR intensities for emission of a three-layered spherical structure;

- the relation between the Einstein coefficient for spontaneous emission and the intensity of the resonant spontaneous emission from an open cavity has been established in the form of Eq. (13);

- the calculations fulfilled in the framework of classical electrodynamics and with the proposed algorithm applying the quantum theory have been shown to agree well with each other and with experimental data.

Properties of optical microsensors of a new generation, exploiting the properties of the evanescent part of the whispering-gallery modes, have been evaluated.

\section{References}

1. A. Rosenberger and J. P. Rezac, Whispering-gallery-mode evanescent-wave microsensor for trace-gas detection // Proc. SPIE, 4265, pp. 102-112 (2001).

2. F. Vollmer, D. Braun, A. Libchaber, M. Khoshsima, I. Teraoka, and S. Arnold, Protein detection by optical shift of a resonant microcavity // Appl. Phys. Lett., 80(21), pp. 4057 4059 (2002)

3. A. Serpenguzel, J.C. Swindal, R.K. Chang, and W.P. Acker, Two-dimensional imaging of sprays with fluorescence, lasing, and stimulated Raman scattering // Appl. Opt., 31(18), pp. 3543-3551 (1992)

4. T. Takahashi, K. Fujiwara, S. Matsuo, and H. Misawa, Excitation energy transfer between dye molecules in lasing microparticles // J. Photochem. Photobiol. A, 120(2), pp. 135140 (1999).
5. W. von Klitzing, R. Long, V. S. Ilchenko, J. Hare, and V. Lefevre-Seguin, Tunable whispering gallery modes for spectroscopy and CQED experiments // New J. Phys., 3, pp. 14.1-14.14 (2001)

6. K. Sasaki, H. Fujiwara, and H. Masuhara, Photon tunneling from an optically manipulated microsphere to a surface by lasing spectral analysis // Appl. Phys. Lett., 70(20), pp. 26472649 (1997).

7. V.V. Datsyuk and I.A. Izmailov, Optics of microdroplets // Phys.-Uspekhi, 44(10), pp. 1061-1073 (2001).

8. A.N. Oraevsky, Waves of whispering gallery // Kvant. Elektron, 32(5), pp. 377-400 (2002).

9. P. Chylek, H.-B. Lin, J.D. Eversole, and A.J. Campillo, Absorption effects on microdroplet resonant emission structure // Opt. Lett., 16(22), pp. 1723-1725, (1991).

10. V.V. Datsyuk, Gain effects on microsphere resonant emission structures // J. Opt. Soc. Am. B, 19(1), pp. 142-147 (2002).

11. V.V. Datsyuk, Spontaneous radiation of molecules in open cavities // JETP Letters, 75(8), pp. 368-372 (2002).

12. L. Knoll, S. Scheel, and D.-G. Welsch, Coherence and statistics of photons and atoms // arXiv: quant-ph/0006121 (2000).

13. H.T. Dung, L. Knoll, and D.-G. Welsch, Spontaneous decay in the presence of dispersing and absorbing bodies: General theory and application to a spherical cavity // Phys. Rev. A, 62(5), pp. 053804-1-053804-13 (2000).

14. H.T. Dung, L. Knoll, and D.-G. Welsch, Decay of an excited atom near an absorbing microsphere // Phys. Rev. A, 64(1), pp. 013804-1-013804-15 (2001).

15. R. T. Wang and H. C. van de Hulst, Rainbows: Mie computations and the Airy approximation // Appl. Opt., 30(1), pp. 106117 (1991).

16. F. V. Bunkin and A. N. Oraevsky, On spontaneous emission of molecule inside cavity // Izv. Vyssh. Uchebn. Zaved., Radiofiz., 2(2), pp. 181-186 (1959).

17. S.C. Ching, H.M. Lai, and K. Young, Dielectric microspheres as optical cavities: Einstein A and B coefficients and level shift // J. Opt. Soc. Amer. B, 4(12), pp. 2004-2009 (1987).

18. H. M. Lai, P. T. Leung, and K. Young, Electromagnetic decay into a narrow resonance in an optical cavity // Phys. Rev. A, 37(5), pp. 1597-1606 (1988).

19. A.J. Campillo, J.D. Eversole, and H.-B. Lin, Cavity quantum electrodynamic enhancement of spontaneous and stimulated emission in microdroplets // Mod. Phys. Lett., 6(8), pp. 447457 (1992)

20. H.-B. Lin, J.D. Eversole, C.D.Meritt, and A.J.Campillo, Cavity-modified spontaneous-emission rates in liquid microdroplets // Phys.Rev. A, 45(9), pp. 6756-6760 (1992).

21. Y. Xu, R. K. Lee, and A. Yariv, Quantum analysis and the classical analysis of spontaneous emission in a microcavity // Phys. Rev. A, 61(3), 033807-1-033807-13 (2000).

22. M. L. Gorodetsky, A. D. Pryamikov, V. S. Ilchenko, Rayleigh scattering in high- $Q$ microspheres // J. Opt. Soc. Am. B, 17(6), pp. 1051- 1057 (2000). 